\title{
Decentralised MPC based on a Graph Partitioning Approach applied to the Barcelona Drinking Water Network ${ }^{\star}$
}

\author{
Carlos Ocampo-Martinez Vicenç Puig Samuele Bovo \\ Institut de Robòtica i Informàtica Industrial (CSIC-UPC) \\ Llorens $i$ Artigas 4-6, 08028 Barcelona, Spain \\ \{ cocampo, vpuig, sbovo\}@iri.upc. edu
}

\begin{abstract}
In this paper, the application of decentralised model predictive control (DMPC) over a partitioned model of the Barcelona drinking water network (DWN) is proposed. The system decomposition is based on graph partitioning theory, which considers the graph representation of the DWN topology. Once obtained, this system decomposition yields to some bidirectional flows of information between the MPC controllers. Hence, the proposed DMPC strategy copes with this feature, resulting in a hierarchical-like scheme. Results obtained when used selected simulation scenarios show the effectiveness of the control strategy in terms of system modularity, reduced computational burden and, at the same time, the admissible loss of performance in contrast to a centralised MPC (CMPC) strategy.
\end{abstract}

Keywords: Model predictive control, large-scale networked systems, systems partitioning, hierarchical control, decentralised control, drinking water networks

\section{INTRODUCTION}

Drinking water management in urban areas is a subject of increasing concern as cities grow. Limited water supplies, conservation and sustainability policies, as well as the infrastructure complexity for meeting consumer demands with appropriate flow pressure and quality levels make water management a challenging problem. Many modern water systems are operated through centralised telecontrol systems. In most cases, network operation is carried out using empirical rules and "historic" strategies, which were result of years of operational experience and empirical results. While these may generally be adequate, the best operational policies may be very complex to be determined in large-scale interconnected networks. Thus, decisionsupport systems for operational control, which are based on mathematical models of network operation and optimal control techniques, provide useful guidance for efficient management of water networks.

Model-based Predictive Control (MPC) has been proved to be one of the advanced control techniques, widely accepted for the operational control of water systems (Brdys and Ulanicki, 1994; Marinaki and Papageorgiou, 2005; Overloop, 2006). The main reason is due to once obtained the network dynamical model, the MPC design just consists in expressing the desired performance specifications through different control objectives and constraints on system variables (e.g., minima/maxima of selected process variables and/or their rates of change), which are necessary

\footnotetext{
* This work has been supported by Spanish research project WATMAN (CICYT DPI2009-13744) of the Science and Technology Ministry, the Juan de la Cierva Research Programme (ref. JCI-20082438), the DGR of Generalitat de Catalunya (SAC group Ref. 2009/SGR/1491) and the EU project WIDE (FP7-IST-224168).
}

to ensure process safety and asset health. The rest of the MPC design is automatic: the given model, constraints, and weights define an optimal control problem over a finite time horizon in the future (for this reason the approach is said predictive).

Nevertheless, the main hurdle for MPC control, as any other control technique, when applied to large-scale networks in a centralised way, is the non-scalability. The reason is that a huge control model is needed, being difficult to maintain/update and which needs to be rebuilt on every change in the system configuration as for example, when some part of the system should be stopped because of maintenance actions or malfunctions. Subsequently, a model change would require re-tuning the centralised controller. It is obvious that the cost of setting up and maintaining the monolithic solution of the control problem is prohibitive. A way of circumventing these issues might be by looking into decentralised MPC (DMPC) or distributed MPC techniques, where networked local MPC controllers are in charge of the control of part of the entire system.

The success of centralised MPC (CMPC) drives now a new interest in this old area of distributed control, becoming DMPC in one of the hottest topics in control during the early $21^{\text {st }}$ century. Few works have been recently published in this area; see, e.g., Keviczky et al. (2006); Rawlings and Stewart (2008); Negenborn et al. (2008); Venkat et al. (2008); Scattolini (2009), among others. However, there is a prior problem to be solved: the system decomposition into subsystems. The importance of this issue has already been noticed in classic-control books addressing the decentralised control of large-scale systems (LSS); see, e.g., Šiljak (1991) or Lunze (1992). These references propose some approaches for dealing with the decomposition of 
dynamical networked systems under certain assumptions, which are related to the level of coupling of the constitutive elements belonging to the considered LSS.

The main contribution of this paper consists in presenting the application of a hierachical-like DMPC approach to the Barcelona drinking water network (DWN). The aim is to show that DMPC reduces the computational burden with respect to the centralised counterpart, but still maintaining a convenient level of suboptimality with respect to the desired control objectives. Moreover, important features such as the system modularity are presented in a decentralised scheme. The advantage of the hierarchicallike DMPC approach is the simplicity of its implementation given the absence of negotiations among controllers. To apply the proposed DMPC approach, the network is decomposed into subsystems using a novel automatic decomposition algorithm reported in Ocampo-Martinez et al. (2011), which is based on graph partitioning.

The paper is structured as follows: Section 2 describes the case study considered in the paper. Section 3 briefly introduces the partitioning approach for dynamical systems used with the case study of this paper. Section 4 presents and discusses the hierarchical-like DMPC strategy applied to the case study. Section 5 discusses the main results derived from the application of the proposed control approach over the considered case study. Finally, conclusions and directions for further research are reported in Section 6.

\section{CASE-STUDY DESCRIPTION}

\subsection{System Description}

The Barcelona DWN, managed by Aguas de Barcelona, S.A. (AGBAR), not only supplies drinking water to Barcelona city but also to the metropolitan area. The sources of water are the Ter and Llobregat rivers, which are regulated at their head by some dams with an overall capacity of 600 cubic hectometres. Currently, there are four drinking water treatment plants (WTP)and several underground sources (wells) that can provide water through pumping stations. Those different water sources currently provide a flow of around $7 \mathrm{~m}^{3} / \mathrm{s}$. The water flow from each source is limited, what implies different water prices depending on water treatments and legal extraction canons.

The Barcelona DWN is structurally organised in two layers. The upper layer, named as transport network, links the water treatment plants with the reservoirs distributed all over the city. The lower layer, named distribution network is sectorised in subnetworks. Each subnetwork links a reservoir with each consumer. This paper is focused on the transport network. Thus, each subnetwork of the distribution network is modelled as a demand sector. The demand of each sector is characterised by a demand pattern, which can be predicted by using a time-series model (Quevedo et al., 2010). The control system of the transport network is also organised in two layers (see Figure 1). The upper layer is in charge of the global control of the network, establishing the set-points of the regulatory controllers at the lower layer. Regulatory controllers are of PID type, while the supervisory layer controller is of MPC

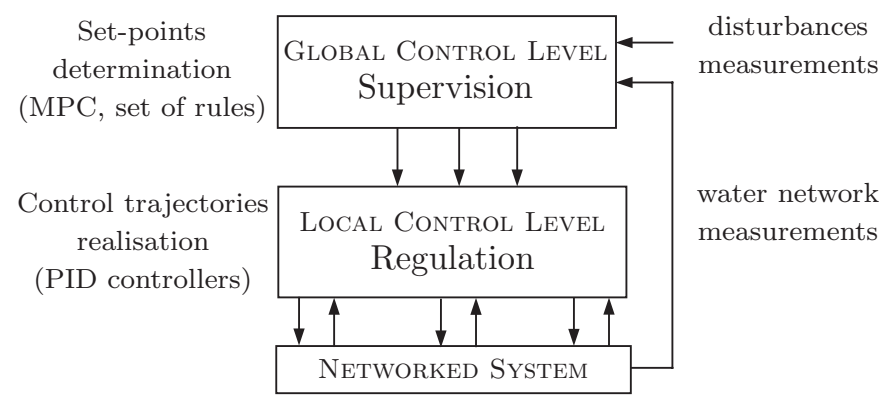

Fig. 1. Hierarchical structure for RTC system

type. Regulatory controllers hide the network non-linear behaviour to the supervisory controller. This fact allows the MPC supervisory controller to use a control-oriented linear model.

\subsection{System Management Criteria}

AGBAR has provided the management policies for the Barcelona DWN, given their knowledge of the system. These management criteria are explained below.

Minimising water production and transport costs The main economic costs associated with drinking water production (treatment) are due to chemicals, legal canons, and electricity costs. The corresponding performance index to be minimised is expressed as

$$
f_{1}(t)=W_{e}\left(\alpha_{1}+\alpha_{2}(t)\right) u(t)
$$

where $u$ denotes the manipulated flows through the system actuators, $\alpha_{1}$ corresponds to a known vector related to the economic cost of the water according to the selected source (treatment plant, dwell, etc.), and $\alpha_{2}(t)$ is associated with the economic cost of the flow through certain actuators (pumps only) and their control cost (pumping). Variable $t$ denotes the discrete time. Note the time variance of $\alpha_{2}$ due to the fact that pumping efforts have different values according to the time of the day (electricity costs). The weight $W_{e}$ is the penalty associated with economic costs with respect to other objectives included in the MPC optimisation problem. Also notice the linear nature of (1) given by the unidirectional feature of all the manipulated flows.

Safety storage term The satisfaction of water demands should be fulfilled at every time instant. However, some risk prevention mechanisms should be introduced in the tank management so that, additionally, the stored volume is preferably maintained around a given safety value in case of emergency, and to guarantee future water availability in case of inaccurate demand forecasts. A quadratic expression for this concept is used and written as follows:

$$
f_{2}(t)=\left(x(t)-\beta x^{\max }\right)^{T} W_{x}\left(x(t)-\beta x^{\max }\right),
$$

where $x$ denotes the water volumes at network tanks, $\beta$ is a term which determines the safety volume to be considered for the control law computation and matrix $W_{x}$ defines the weight of the objective in the cost function. This term prevents the controller from keeping the lowest possible water volumes in the tanks, which would reduce the robustness to demand forecast inaccuracy. 
Smoothness of control actions To smooth out the control action of MPC, the following third term is included in the objective function to penalise variations $\Delta u(t)=u(t)-$ $u(t-1)$ of the control signal between consecutive sampling intervals:

$$
f_{3}(t)=\Delta u(t)^{T} W_{\Delta u} \Delta u(t)
$$

where $W_{\Delta u}$ is a $m \times m$ weight matrix.

\subsection{Control-oriented Modelling}

Control-oriented modelling principles for DWNs have been widely presented in the literature, see Brdys and Ulanicki (1994); Ocampo-Martinez et al. (2009). In order to obtain a control-oriented model of the DWN, the constitutive network elements as well as their basic relationships should be discussed. The reader is referred to the aforementioned references for further details of DWN modelling and specific insights related to the case study of this paper.

Consider the main physical constraints of the DWN given by the variables related to the tank volumes and manipulated flows. In the case of tank volumes, the physical constraint related to the range of volume capacities for the $i$-th tank is expressed as

$$
x_{i}^{\min } \leq x_{i}(t) \leq x_{i}^{\max }, \quad \forall t,
$$

where $x_{i}^{\min }$ and $x_{i}^{\max }$ denote the minimum and maximum volume capacity, respectively, given in $\mathrm{m}^{3}$. On the other hand, physical constraints related to manipulated flows through the system actuators are expressed as

$$
u_{i}^{\min } \leq u_{i}(t) \leq u_{i}^{\max }, \quad \forall t,
$$

where $u_{i}^{\min }$ and $u_{i}^{\max }$ denote the minimum and the maximum flow capacity, respectively, given in $\mathrm{m}^{3} / \mathrm{s}$.

By considering the mass balance in tanks, the controloriented model of the DWN in discrete-time state-space form can be written as

$$
x(t+1)=A x(t)+B u(t)+B_{p} d(t),
$$

where $x \in \mathbb{R}^{n}$ is the state vector corresponding to the water volumes of the $n$ tanks, $u \in \mathbb{R}^{m}$ represents the vector of manipulated flows through the $m$ actuators (pumps and valves), and $d \in \mathbb{R}^{p}$ corresponds to the vector of the $p$ water demands (sectors of consume). $A$, $B$, and $B_{p}$ are system matrices of suitable dimensions. Demands are forecasted in the prediction horizon using time series models. Thus, $d$ is a known vector containing the forecasted disturbances affecting the system. By also including static relations at network nodes, model (6) can be further rewritten as

$$
\begin{aligned}
x(t+1) & =A x(t)+\Gamma v(t), \\
E_{1} v(t) & =E_{2},
\end{aligned}
$$

where $\Gamma=\left[\begin{array}{ll}B & B_{p}\end{array}\right], v(t)=\left[\begin{array}{ll}u(t)^{T} & d(t)^{T}\end{array}\right]^{T}$, and $E_{1}$, $E_{2}$ are matrices of suitable dimensions dictated by the network topology.

The Barcelona DWN model (7) contains a total amount of 67 tanks and 121 actuators, these latter divided in 46 pumps and 75 valves. Moreover, the network has 88 demand sectors and 16 water nodes. Both the demand episodes and the network calibration/simulation setup are provided by AGBAR. Figure 2 (further below) depicts the considered network.

\section{PARTITIONING APPROACH}

\subsection{General Algorithm}

The partitioning algorithm related to the case study of this paper requires to represent the dynamical system as a graph, which can be obtained from the system structure, (Šiljak, 1991). Thus, the desired partitioning of the dynamical system is translated to a partition of a directed graph. The current version of the algorithm is though to be used off-line, i.e., the partitioning of the system is not carried out on-line. A further improvement might be to adapt the proposed algorithm such that the partitioning could be done on-line when some structural change of the network occurs. The objective of the partitioning algorithm is to obtain a graph decomposition as less interconnected as possible, with similar number of vertices for each subgraph.

The main procedures/routines immersed in the algorithm are briefly described below. For further and detailed information about this partitioning algorithm, the reader is referred to Ocampo-Martinez et al. (2011).

Start up This procedure requires the definition of the graph, i.e., the incidence matrix $I_{M}$, which describes the connections between the graph vertices, their directionality and, in some cases, the weight of each edge.

Preliminary partitioning This procedure performs a preliminary automatic partitioning of the graph, defining vertices with high amount of connections and associating the other vertices by zones. This routine highlights the subgraphs of higher connectivity. From this procedure, a set of $k$ subsystems is determined for a subsequent refining according to given criteria.

Uncoarsening - Internal balance This procedure aims at the reduction of the number of subgraphs, trying to achieve similar internal weights for all of them, where the internal weight is defined as the number of vertices of each subgraph. The way of achieving this objective consists in merging subgraphs under a certain conditions and values of their indices.

Refining - External balance This procedure aims at the minimisation for each subgraph of the number of edges with endpoints in different subgraphs. To achieve this goal, this routine balances the vertex internal degree (VID) and the vertex external degree (VED) for each vertex of each subgraph, where the VID denotes the number of vertex connections with vertices belonging to the same subgraph, while VED is defined as the number of vertex connections with vertices of different subgraphs.

Auxiliary Routines Even the execution of the explained routines yields an automatic partitioning of a given graph, it does not imply that the resultant set of subgraphs follows the pre-established requirements. In this sense, complementary routines can be useful for improving the partitioning process according to the considered application. Here, the following auxiliary routines are taken into account:

- Pre-filtering: this routine virtually merges those vertices that shares its unique edge, creating supranodes. 
Table 1. Dimensions of the DWN subsystems

\begin{tabular}{ccccc}
\hline Subsystem & Tanks & Actuators & Demands & Nodes \\
\hline 1 & 13 & 36 & 20 & 5 \\
2 & 11 & 11 & 11 & 0 \\
3 & 13 & 22 & 20 & 3 \\
4 & 9 & 16 & 12 & 2 \\
5 & 6 & 10 & 8 & 2 \\
6 & 15 & 26 & 17 & 3 \\
\hline Total & 67 & 121 & 88 & 15 \\
\hline
\end{tabular}

- Post-filtering: this routine allows to relax the internal balance of the subgraphs by adding certain tolerance $\delta$ in the criteria of the uncoarsening routine. This relaxation allows to have less subgraphs but with higher complexity and internal weight.

- Anti-oscillation: this routine avoids the possible infinite loop defined by merging/split subsystems when the refining routine is run. Therefore, the parameter $\rho$ is set as the maximum number of iterations that the refining routine is executed.

The Complete Algorithm It consists of the collection of the procedures/routines mentioned before. Hence, applying this algorithm to the graph associated to a given dynamical system, the expected result consists of a set $P$ of subgraphs which determines a particular system decomposition.

\subsection{Case Study Partitioning}

This section presents the results of the application of the decomposition algorithm proposed in Ocampo-Martinez et al. (2011) for the partitioning of the Barcelona DWN into compositional subsystems. The graph of the Barcelona DWN has been derived from its topological structure under the following considerations:

- every tank, sector of consume, water source and node is considered as a vertex of the graph;

- every pump, valve and link with a sector of consume is considered as a graph edge.

Results have been obtained setting $\rho=250$ for the anti-oscillation routine and $\delta=2$ for the post-filtering routine, what produces a partitioning with six subgraphs. Each subgraph ofthis decomposition corresponds to one subsystem of the Barcelona DWN with the number of elements presented in Table 1. Figure 2 shows, in different colours, the obtained subsystems of Barcelona DWN. Figure 3 schematically depicts the resultant subsystems and the sets of shared links between the network subsystems including their directions.

\section{CONTROL STRATEGY APPROACH}

Using the Barcelona DWN decomposition considering the partitioning algorithm in Section 3, a DMPC strategy is implemented in order to manage the networked system. This DMPC strategy considers

- the dynamical system model (7) split in 6 subsystems;

- the physical constraints (4)-(5) for each subsystem;

- a demand forecasting algorithm (taken from OcampoMartinez et al. (2009); Quevedo et al. (2010)); and

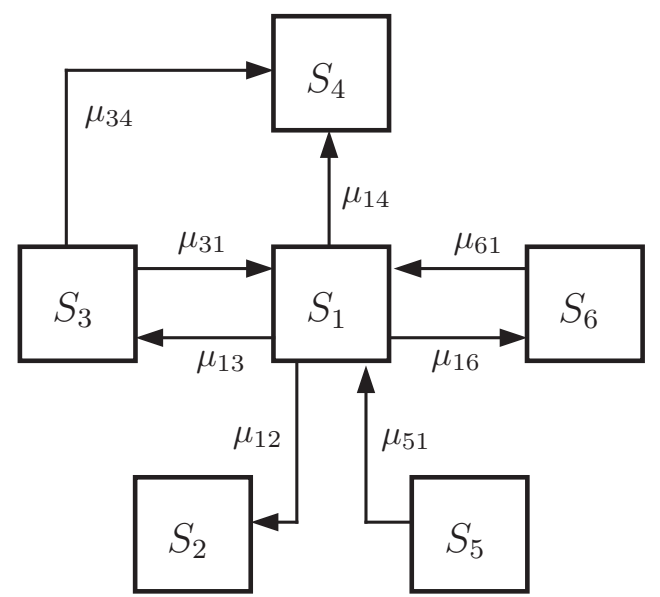

Fig. 3. Network subsystems $S_{i}$ and their sets of shared connections $\mu_{i j}$

- a multi-objective cost function, expressed by using (1), (2), and (3) as

$$
J(t)=\sum_{i=0}^{H_{u}-1} f_{1}(t+i \mid t)+\sum_{i=1}^{H_{p}} f_{2}(t+i \mid t)+\sum_{i=0}^{H_{u}-1} f_{3}(t+i \mid t),
$$

where $H_{p}$ and $H_{u}$ correspond to the prediction and control horizons, respectively, index $t$ represents the current time instant while index $i$ represents the predicted time along $H_{p}$. In this paper, the prediction horizon is related to the 24 -hours demand seasonality. Moreover, $H_{u}=H_{p}$, following the criterion of the DWN management company.

According to Šiljak (1991), the pure hierarchical control scheme determines a sequence of information distribution among the subsystems, where top-down communication is available from upper to lower level of the hierarchy. Note that, despite the subsystems coupling (given by the shared links), the main feature of the pure hierarchical control approach relies on the unidirectionality of the information flow between controllers.

Looking at Figure 4, where the directions of the sets of shared links are graphically shown, it is possible to realise that two of those sets, denoted by $\mu_{13}$ and $\mu_{16}$ (red dashed lines in the figure), break the mentioned unidirectional flow between MPC controllers. This fact implies that the standard hierarchical control scheme for partitioned LSS cannot be straight applied. To solve this situation and design a DMPC law, a hierarchical-like DMPC approach is proposed and conveniently implemented. This strategy follows the hierarchical control philosophy and the sequential way of solving the optimisation subproblems of the corresponding MPC controllers but also considering the appearance of bidirectional information flows. For this purpose, additional constraints and heuristics are taken into account in order to cope with the feature of having the double direction in the flow of information between some of the controllers. In particular, Figure 4 shows the considered hierarchy for the case study of this paper, where controllers at the first level of hierarchy determine the values of variables shared with controllers in lower levels. Notice that Figure 4 also shows why the pure hierarchi- 


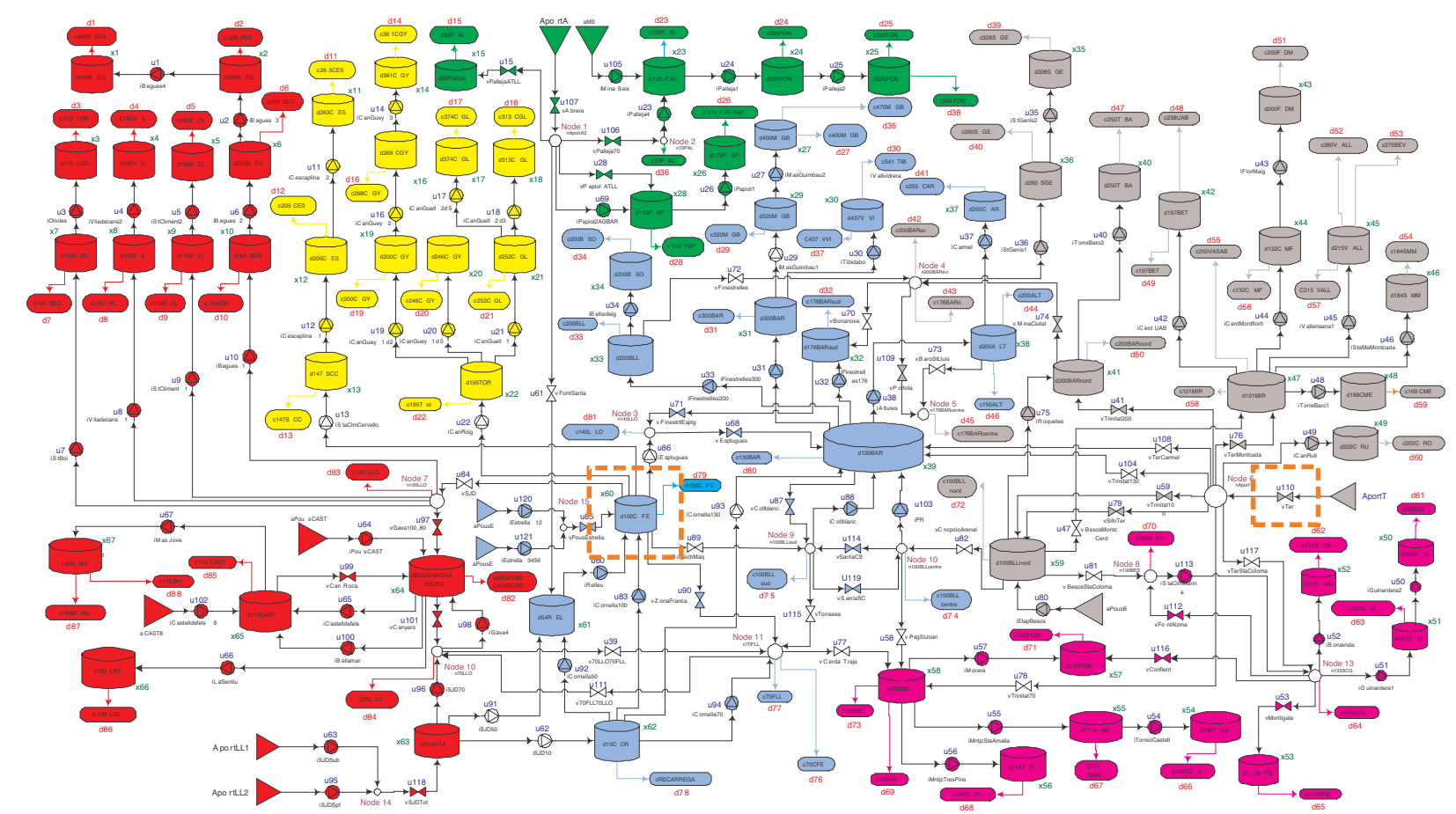

Fig. 2. Definitive partition of the Barcelona DWN. The key elements are properly featured

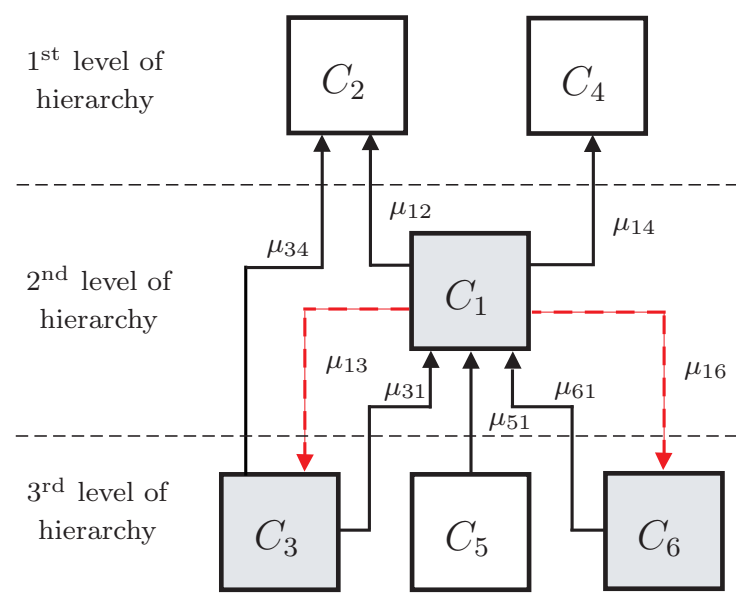

Fig. 4. Hierarchy of MPC controllers $C_{i}$. Their solution sequence is top-down

cal control approach cannot be employed since the MPC controller $C_{1}$ shares bidirectional information with $C_{3}$ and $C_{6}$.

Hence, in order to explain the implementation of the solution sequence for the considered hierarchical-like DMPC strategy, denote $C_{i}$ as the MPC controller related to the subsystem $S_{i}$ (for $i \in\{1, \ldots, 6\}$ ), and $\mu_{i j}$ as the set of control actions $u$ (manipulated flows, see (7)) going from $S_{i}$ to $S_{j}$ (for $j \in\{1, \ldots, 6\}, i \neq j$ ). Notice that $\mu_{i j}$ not only contains values of each component at time $t$ but also all values over $H_{u}$, i.e., if $\mu_{i j}=\left\{u_{a}, u_{b}, \ldots\right\}$, then ${ }^{1}$

\footnotetext{
1 With some abuse of the notation, the elements of vector $u$ are denoted with the corresponding discrete-time dependence in order to differentiate the vector from its components.
}

$$
\begin{aligned}
& u_{a} \triangleq\left[\begin{array}{llll}
u_{a}(t \mid t) & u_{a}(t+1 \mid t) & \ldots & u_{a}\left(t+H_{u}-1 \mid t\right)
\end{array}\right]^{T}, \\
& u_{b} \triangleq\left[\begin{array}{llll}
u_{b}(t \mid t) & u_{b}(t+1 \mid t) & \ldots & u_{b}\left(t+H_{u}-1 \mid t\right)
\end{array}\right]^{T}
\end{aligned}
$$

with $u_{a}(t+i \mid t)$ denoting the value of $u_{a}$ at time $t+i$ (over the control horizon) given $t$. Therefore, the solution sequence of the described hierarchical-like control problem for the complete Barcelona DWN at each iteration $t \in \mathbb{Z}_{\geq 1}$ is the following:

- $C_{4}$ computes the control actions of $S_{4}$ and sets $\mu_{14}$ and $\mu_{34}$.

- In parallel, $C_{2}$ computes the control actions of $S_{2}$ and the set $\mu_{12}$.

- $C_{1}$ computes the control actions of $S_{1}$ and sets $\mu_{31}, \mu_{51}$, and $\mu_{61}$. Sets $\mu_{12}, \mu_{13}, \mu_{14}$, and $\mu_{16}$ are considered as sets of virtual demands ${ }^{2}$ within the controller $C_{1}$.

- $C_{5}$ computes the control actions of $S_{5}$ considering $\mu_{51}$ as a set of virtual demands.

- $C_{3}$ computes the control actions of $S_{3}$ considering $\mu_{31}$ and $\mu_{34}$ as sets of virtual demands. $C_{3}$ also computes the set $\mu_{13}$ to be used as a set of virtual demands for $C_{1}$ at iteration $t+1$.

- $C_{6}$ computes the control actions of $S_{6}$ considering $\mu_{61}$ as a set of virtual demands. $C_{6}$ also computes $\mu_{16}$ to

${ }^{2}$ Consider two subsystems $S_{1}$ and $S_{2}$, which share a set of manipulated flows $\mu_{12}$. According to the notation employed in the paper, those flows come from $S_{1}$ to $S_{2}$. If the solution sequence of optimisation subrproblems - defined by the pre-established hierarchical order - determines that $\mu_{12}$ is computed by the MPC controller of $S_{1}$, then flows in $\mu_{12}$ are considered as virtual demands in the controller related to $S_{2}$ since their value are now imposed in the same way as the water demands. 
be used as a set of virtual demands for $C_{1}$ at iteration $t+1$.

Remark 4.1. Notice that in the proposed DMPC scheme, at the first iteration $(t=1)$, the initial values of the control actions belonging to sets $\mu_{13}$ and $\mu_{16}$ are not available. Those values can be obtained by solving a constraint satisfaction problem (CSP) defined by the models and constraints of subsystems $S_{1}, S_{3}$ and $S_{6}$ (shaded blocks in Figure 4) through the algorithm proposed in Jaulin et al. (2001). The solution of this CSP provides feasible control actions for sets $\mu_{13}$ and $\mu_{16}$, what allows starting the solution sequence described above. For subsequent iterations, values of $\mu_{13}$ and $\mu_{16}$ take values computed by $C_{3}$ and $C_{6}$, respectively, in the previous iteration, i.e., the elements belonging to those sets at time $t$ are now assigned as (see (9))

$$
u=\left[\begin{array}{c}
u(t+1 \mid t-1) \\
\vdots \\
u\left(t+H_{u}-1 \mid t-1\right) \\
u\left(t+H_{u}-1 \mid t-1\right)
\end{array}\right]
$$

\section{RESULTS}

The results obtained by using the proposed DMPC strategy are compared with those obtained employing a CMPC approach. Two scenarios corresponding to different prioritisations of the control objectives have been considered for the performance comparison of the MPC strategies:

- Scenario 1: $\Psi=(0.7,0.2,0.1)$,

- Scenario 2: $\Psi=(0.6,0.2,0.2)$,

where $\Psi=\left(\psi_{e}, \psi_{x}, \psi_{\Delta t}\right)$ represents the 3 -tuple of weights associated to the weight matrices $W_{e} \triangleq \psi_{e} I, W_{x} \triangleq \psi_{x} I$, $W_{\Delta u} \triangleq \psi_{\Delta u} I$ used at the normalised functions (1), (2), and (3), respectively ${ }^{3}$. Notice that, given the employed normalisation of the control objective terms in the cost function (8), the sum of $\psi_{i}$, for $i \in\{e, x, \Delta u\}$, should be 1. The tuning scenarios are chosen in such a way that the highest priority objective is the economic cost (see Section 2.2), which should be minimised while maintaining a similar rate of the safety volume and control action smoothness terms.

All results have been obtained considering real demand episodes of four days (with 1 hour sampling time), with initial volumes in tanks set to $40 \%$ of their maximum volume, $H_{p}=H_{u}=24$, and the safety volume parameter $\beta$ set to 0.8 . All simulations have been performed in MATLAB ${ }^{\circledR}$ 7.1 implementations running on an Intel ${ }^{\circledR} \mathrm{Core}^{\mathrm{TM}} 2,2.4$ $\mathrm{GHz}$ machine with 4Gb RAM.

Table 2 summarises the obtained control results in terms of performance (economical cost) and computational burden over four days. The indices representing costs are given in economic units (e.u.) instead of Euro due to confidentiality restrictions. Computation times are given in seconds.

From Table 2, it can be noticed the loss of performance when using the DMPC approach, introducing a sub-optimality of about $15 \%$. This loss of performance is obtained because the DMPC strategy does not take into account in a proper way the water costs related to

\footnotetext{
3 Matrix $I$ denotes the identity matrix of suitable dimensions.
}

Table 2. Computation time and performance comparisons

\begin{tabular}{ccccc}
\hline \multirow{2}{*}{ IndEx } & \multicolumn{2}{c}{ Scenario 1 } & \multicolumn{2}{c}{ Scenario 2 } \\
& CMPC & DMPC & CMPC & DMPC \\
\hline Water Cost & 138.37 & 189.45 & 137.05 & 188.81 \\
Electric Cost & 92.73 & 68.44 & 87.43 & 69.91 \\
Total Cost & 231.10 & 257.89 & 224.48 & 258.72 \\
CPU time & 1143 & 537 & 1127 & 560 \\
\hline
\end{tabular}

external water sources since it is a global objective. On the other hand, DMPC controllers are mainly focused on the reduction of pumping costs (local objective) within each subsystem. By contrast, the information of water costs is properly managed for the CMPC controller by optimising it but at the price of moving more water inside the network. This leads to an increment in the electric costs (the water transportation cost) when CMPC controller is used. Therefore, despite the DMPC approach inevitably leads to a loss of performance, the benefits in terms of time and computational burden are significant enough, what makes it suitable for real-time implementation purposes. Notice that in this particular application, the CMPC could also satisfy the real-time constraint since the control sampling time is 1 hour. So, the main motivation for using DMPC in this application would not be the improvement in computation but the scalability and the potential adaptability easiness facing network changes that could occur.

Regarding the dynamical closed loop behaviour of the network, Figures 5 and 6 show the flow through a water supply valve and the volume of a key tank, respectively (see those elements highlighted in Figure 2), for both predictive control strategies. Notice in Figure 5 that the behaviour of the volume is qualitatively equivalent for both strategies since the filling and emptying processes of the associated tank follows the demand evolution. On the other hand, notice that in Figure 6 the water inflow from this source is greater when DMPC is implemented. As discussed before, DMPC strategy makes that the water of each subsystem is supplied by its own sources, reducing the water transportation within the network. Hence, this source is providing almost all the water that this subsystem needs in contrast to the CMPC case, where the water was moved from other network locations (due to its cheaper price). This fact explains why the DMPC strategy yields a suboptimal solution compared with the CMPC counterpart. This degree of suboptimality is inherent to the followed hierarchal approach since each controller is mainly focused on optimising the control objectives related to the subnetwork that is controlling. A further improvement of the proposed approach would consists in adding some improved coordination mechanism in the control objective of each local MPC controller. This feature allows to take into account the economic costs in a global way.

\section{CONCLUSIONS}

This paper has presented the application of a proposed hierachical-like DMPC approach to the Barcelona DWN. To apply this control strategy, the network is partitioned into subsystems by using a novel automatic decomposition algorithm based on graph partitioning. The hierarchical- 


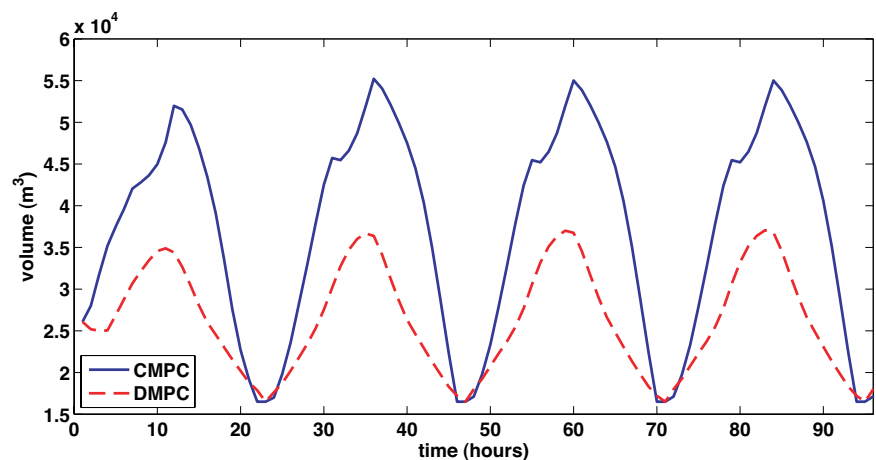

Fig. 5. Resultant volume related to a key tank of the DNW

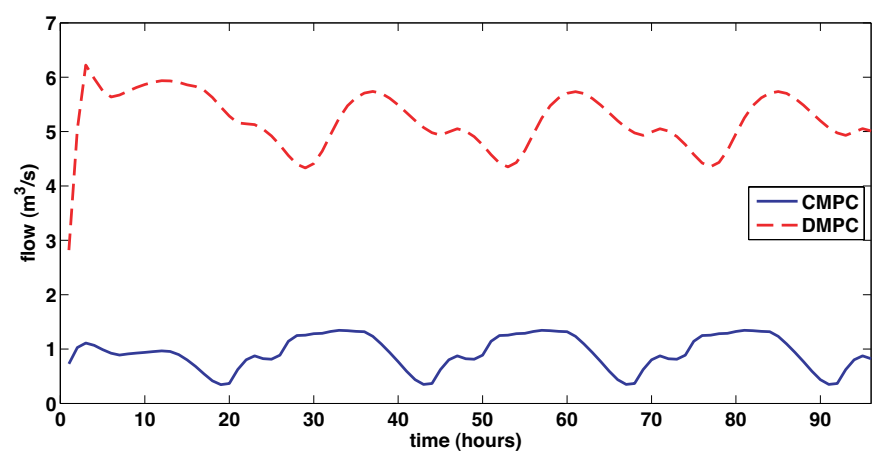

Fig. 6. Computed flow related to a supply water valve

like DMPC scheme should be considered since the resultant DWN decomposition determines a set of subsystems that cannot be grouped in different levels such that the flow of information is unidirectional from clusters at higher hierarchical levels towards clusters at lower levels. This fact implies that a pure hierarchical DMPC approach cannot be considered. A comparison with a CMPC approach show that the level of sub-optimality in economic costs is acceptable considering the resultant reduction in computational burden. As future research, the proposed hierarchical-like approach, which addresses the loops between levels in a heuristic way, should be further investigated in order to evaluate the introduced degree of suboptimality as well as how feasibility and stability features are preserved.

\section{REFERENCES}

M. Brdys and B. Ulanicki. Operational Control of Water Systems: Structures, algorithms and applications. Prentice Hall International, UK, 1994.

L. Jaulin, M. Kieffer, O. Didrit, and E. Walter. Applied Interval Analysis, with Examples in Parameter and State Estimation, Robust Control and Robotics. SpringerVerlag, London, 2001.

T. Keviczky, F. Borrelli, and G.J. Balas. Decentralized receding horizon control for large scale dynamically decoupled systems. Automatica, 42(12):2105-2115, December 2006.

J. Lunze. Feedback Control of Large-Scale Systems. Prentice Hall, Great Britain, 1992.

M. Marinaki and M. Papageorgiou. Optimal Real-time Control of Sewer Networks. Springer, Secaucus, NJ (USA), 2005.
R.R. Negenborn, B. De Schutter, and J. Hellendoorn. Multi-agent model predictive control for transportation networks: Serial vs. parallel schemes. Engineering Applications of Artificial Intelligence, 21(3):353-366, April 2008.

C. Ocampo-Martinez, V. Puig, G. Cembrano, R. Creus, and M. Minoves. Improving water management efficiency by using optimization-based control strategies: the Barcelona case study. Water Science \&6 Technology: Water supply, 9(5):565-575, 2009.

C. Ocampo-Martinez, S. Bovo, and V. Puig. Partitioning approach oriented to the decentralised predictive control of large-scale systems. Journal of Process Control, In Press, 2011. doi: DOI: 10.1016/j.jprocont.2010.12.005.

P.J. Van Overloop. Model Predictive Control on Open Water Systems. Delft University Press, Delft, The Netherlands, 2006.

J. Quevedo, V. Puig, G. Cembrano, and J. Blanch. Validation and reconstruction of flow meter data in the Barcelona water distribution network. Control Engineering Practice, 11(6):640-651, June 2010.

J. B. Rawlings and B. T. Stewart. Coordinating multiple optimization-based controllers: New opportunities and challanges. Journal of Process Control, 18(9):839-845, October 2008.

R. Scattolini. Architectures for distributed and hierarchical Model Predictive Control: A review. Journal of Process Control, 19(5):723-731, May 2009.

D.D. Šiljak. Decentralized control of complex systems. Academic Press, 1991.

A. N. Venkat, I. A. Hiskens, J. B. Rawlings, and S. J. Wright. Distributed MPC strategies with application to power system automatic generation control. IEEE Tran on Control Systems Technology, 16(6):1192-1206, November 2008. 